ECT. If anything, these results are somewhat worse than the observations of Pippard, possibly because of the effect of being observed upon the practitioners.

It is apparent that extremely junior psychiatrists will be required to administer ECT very shortly after taking up post. Up to a third will receive no more instruction than the advice of another junior colleague and this situation is likely to remain unremedied throughout their training. Although a majority will pick up knowledge through journals, textbooks, exam preparation and Journal Clubs, there is scant evidence that this improves technique. There is reason for particular concern in the case of GP trainees who share ECT rotas but do not complete a psychiatric training.

The evidence we present suggests that trainees are strong advocates of the use of ECT and believe it to be effective. Nevertheless, between a fifth and a quarter of those interviewed felt less than competent to give ECT. Ignorance of the apparatus was present in over $\mathbf{4 0} \%$. Regarding actual practice, a minority of trainees alter the dose in a rational manner and over one third respond to a failed stimulation with stimulation at the same settings, whereas increasing the dosage is recommended (Royal College Guidelines, 1989). It is rarer still for trainees to re-stimulate in the event of a brief or partial seizure and all but one considered seizure lengths of less than 25 seconds to be therapeutic, whereas the Royal College guidelines recommend 25 seconds as an "approximate clinical guide". Most trainees are unaware of the Hamilton cuff technique for more accurate timing of seizure length.
The majority of our trainees administered ECT on a rota basis, a practice criticised by Pippard (1992), on the grounds that a trainee rarely sees a patient through an entire course, re-inforcing the "buttonpusher" mentality. However properly ECT may be prescribed, the use of unsupervised and inadequately trained operators must lead to continuing poor practice and reduced therapeutic effectiveness of this powerful treatment.

What of the future? Benbow (1986) has reported on the usefulness of video-taped demonstrations of ECT practice in improving trainees' administration of ECT. Following the report of Pippard (1992), the Royal College of Psychiatrists has commenced an initiative to train consultants and anaesthetists in ECT techniques and theory. Eventually such initiatives will filter down to the training grades. For the moment, our study suggests that training in and supervision of ECT given by junior psychiatrists is at best patchy and, in many instances, seriously deficient.

\section{References}

BENBOW, S. M. (1986) Effect of training on administration of electroconvulsive therapy by junior doctors. Convulsive Therapy, 2, 19-24.

PIPPARD, J. (1992) Audit of electroconvulsive treatment in two NHS regions. British Journal of Psychiatry, 160, $621-637$.

_ \& EllaM, L. (1981) Electroconvulsive treatment in Great Britain. British Journal of Psychiatry, 139, 563-568.

Royal College of Pyschiatrists (1989) The Practical Administration of Electroconvulsive Therapy. London: Gaskell.

\title{
Carbamazepine monitoring
}

\section{Carol Paton, Staff Pharmacist; and Andrew W. Procter, Senior Lecturer, Bexley Hospital, Bexley, Kent DA5 2BW}

\section{Background of the study}

\section{Rationale for monitoring carbamazepine levels}

Carbamazepine fulfils some of the criteria required in order to render a drug suitable for serum level monitoring in that there is poor inter-individual reproducibility of the serum levels achieved with fixed dosage regimes, interbrand differences in bioavailability are known to occur and serum levels can be raised significantly by several commonly prescribed drugs; dextropropoxyphene (a constituent of coprozamol), erythromycin, isoniazid and cimetidine. 
Central to the usefulness of serum level monitoring, however, is the existence of a therapeutic range, as defined by clinical studies. This range has been defined for the anticonvulsant properties of carbamazepine, but not so for its principal uses within psychiatry, i.e. for the treatment and prophylaxis of affective disorders, and in the management of disturbed behaviour.

\section{Pharmacokinetics of carbamazepine and sampling requirements}

The brand of carbamazepine used within Bexley Health Authority is Tegretol. For this formulation, plasma levels peak two hours after oral administration and absorbtion is essentially slow and incomplete (Tegretol-Hospital Pharmacists Guide, 1990). The half-life of carbamazepine following a single oral dose is around $\mathbf{4 8}$ hours. This is reduced significantly to between seven and 24 hours on chronic dosing (i.e. after two to four weeks) due to autoinduction of hepatic metabolising enzymes (Brodie \& Hallworth, 1987), although there is marked individual variability of autoinduction and serum levels obtained with fixed dosage regimes.

Although there is no universally agreed time for collecting blood for assay, it would be logical to assume that trough levels (pre-morning dose) should be measured to determine the relationship with clinical effect, and peak levels (two to four hours postdose) if toxicity is being monitored. This rationale is also applicable when carbamazepine is monitored for indications other than epilepsy (Nolen et al, 1988).

In accordance with the above comments, it would seem reasonable to assume that a trough level taken at least two weeks after the initiation of therapy or change of dose would give the most reliable indicator of the relationship between dose, serum level and clinical effect for any given patient.

The aims of this study were to determine how carbamazepine therapy is monitored locally and how this compares to the known pharmacokinetics of carbamazepine as outlined above.

\section{The study}

Details were collected for all requests for carbamazepine serum level monitoring originating from within the local psychiatric services over three months. Each patient's case-notes were obtained and all information relevant to the assay request was collected: age, reason for request, timing of the sample with respect to the initiation of treatment and change of dose and the last dose administered, any recent missed doses, the reported serum level and the action taken on receiving it. A brief description of the patient's mental state was also recorded in order for investigators to relate any actions taken on receiving the test result to the clinical state of the patient. The date of the previous blood test was also recorded for those patients who were on chronic therapy.

An independent consultant psychiatrist then examined the data and a decision was made, with reasons, as to whether the assay request and the action taken on receiving the results were appropriate or not.

\section{Findings}

\section{Clinical data}

Reason for assay request

During the three months, data were collected for 43 assay requests. Of these, 39 were routine checks usually precipitated by attendance at out-patient clinics or routine physical examinations (long-stay hospital patients), three were made because of suspected toxicity and one as a check of compliance.

\section{Timing of the sample}

The number of days after initiation of treatment or a change in dose before an assay was requested was less than five days for two (5\%) samples, five to 14 days for four $(9 \%)$ samples and longer than 14 days (representing steady state) for $37(86 \%)$ samples.

The length of time between the administration of the last dose and sampling was equal to one dosage interval (representing a trough level) for ten $(23 \%)$ samples. The remaining $33(77 \%)$ samples were taken at various times throughout the dosage interval (representing a random level). Nine (21\%) patients had documented evidence on their in-patient treatment cards that one or more doses had not been administered in the five days immediately preceding the assay.

\section{Action taken on reported assay results}

Two assays from patients with epilepsy were reported as being in the therapeutic range and no change was made to treatment.

The remaining 41 samples were taken from patients with clinical problems other than epilepsy (33 also being random samples), and all fell into the anticonvulsant therapeutic range. The dose was subsequently altered for only one of these patients despite eight being acutely ill with clinical problems other than epilepsy (reported levels at the lower end of the anticonvulsant range) and one being clinically toxic.

\section{Monitoring interval}

Of the 27 patients on chronic therapy, 23 were monitored regularly; ten having had a test within the previous three months, a further nine within the last 
six months and the remaining four within the last nine months.

\section{Comment}

By using the criteria that sampling should take place immediately pre-dose (trough) and at least 14-28 days after initiating therapy/changing dose, in patients who have a clinical condition for which a therapeutic range exists, all but two samples failed to comply, indicating that the doctors' practice in monitoring carbamazepine is not determined by pharmacokinetic principles. This has also been found for lithium monitoring and may be due to poor knowledge of the kinetics which forms the basis of a separate study. This may be improved by formal training and has been adopted locally in the membership course and hospital based teaching sessions.

The assay results, which all fell within the wide (anticonvulsant) range quoted by the laboratory, invariably led to no change in dose despite the clinical presentation of the patient. As already mentioned a therapeutic range (4-12 $\mathrm{mcg} / \mathrm{ml})$ has been defined for carbamazepine in the treatment of epilepsy, although it is not universally accepted. Neurologists have voiced concern that anticonvulsant drugs, with the exception of phenytoin, tend to be overmonitored (Chadwick 1987). The principle of treating the patient and not the serum level is more true when carbamazepine is used in psychiatry as a therapeutic range has not been determined for carbamazepine in the treatment of affective disorders. It should be mentioned in this context that several ranges have been postulated from open trials with small numbers of patients with a large variety of psychiatric problems (Post \& Uhde, 1986). Sampling details are often omitted and many of the trials are methodologically unsound. They also fail to agree on an effective range. The relevance of the anticonvulsant range to other clinical problems must be questioned.
The observation was also made that almost all patients on chronic therapy (more than six months duration) who were monitored at all during the three month observation period, were monitored regularly. None of these patients had any obvious problems relating to their medication. This tends to suggest that assays are requested because of the "need to do something" when a patient is seen rather than for any clinical reason. As well as the rationale behind this practice being questionable, the cost implications in terms of both medical and laboratory staff time should be considered. In view of these factors, this issue merits more widespread discussion and debate to arrive at locally and/or more widely agreed guidelines for the place of therapeutic monitoring of carbamazepine in psychiatry.

\section{Acknowledgements}

We would like to thank the medical and nursing staff of Bexley Hospital and Mrs Patel, principal biochemist at the Brook Hospital, for their co-operation during this study.

\section{References}

Brodie, M. J. \& HAllworth, M. J. (1987) Therapeutic monitoring of carbamazepine. Hospital Update, Jan 87, 57-63.

CHADWICK, D. W. (1987) Overuse of monitoring of blood concentrations of antiepileptic drugs. British Medical Journal, 294, 723-724.

Nolen, W. A., Jansen, G. S. \& Broekman, M. (1988) Measuring plasma levels of carbamazepine; a pharmacokinetic study in patients with affective disorders. Pharmacopsychiatry, 21, 252-254.

Pharmacokinetics and pharmacodynamics. In TegretolHospital Pharmacists Guide. Geigy Pharmaceuticals, 1990.

Post, R. M. \& UHDE, T. W. (1986) Anticonvulsants in nonepileptic psychosis. In Aspects of Epilepsy and Psychiatry (eds. M. R. Trimble and T. G. Bolwig). Chichester: John Wiley. 